\title{
Recent Developments in the Modeling of Heavy Quarkonia
}

\author{
Stanley F. Radford* and Wayne W. Repko ${ }^{\dagger}$ \\ ${ }^{*}$ Department of Physics, Marietta College, Marietta, OH 45750 \\ ${ }^{\dagger}$ Department of Physics and Astronomy, Michigan State University, East Lansing, MI 48824
}

\begin{abstract}
We examine the spectra and radiative decays of the $c \bar{c}$ and $b \bar{b}$ systems using a model which incorporates the complete one-loop spin-dependent perturbative QCD short distance potential, a linear confining term including (spin-dependent) relativistic corrections to order $v^{2} / c^{2}$, and a fully relativistic treatment of the kinetic energy. We compare the predictions of this model to experiments, including states and decays recently measured at Belle, BaBar, CLEO and CDF.
\end{abstract}

\section{INTRODUCTION AND THE POTENTIAL MODEL}

Over the past $25+$ years, potential models have proven valuable in analyzing the spectra and characteristics of heavy quarkonium systems [1, 2, 3, 4, 5, 6, 7]. Motivation for revisiting the potential model interpretation of the $c \bar{c}$ and $b \bar{b}$ systems is provided by recent experimental results:

- The discovery of several expected states in the charmonium spectrum $\left(\eta_{C}\right.$ and $\left.h_{C}\right)$

- The discovery of new states [X(3872), X(3943)], which could be a interpreted as above threshold charmonium levels

- The discovery of the $1^{3} D_{2}$ state of the upsilon system

- The determination of various $E 1$ widths for $c \bar{c}$ and $b \bar{b}$.

Our purpose here is to examine to what extent a semi-relativistic potential model which includes all $v^{2} / c^{2}$ and one-loop QCD corrections can fit the below threshold $c \bar{c}$ and $b \bar{b}$ data and accommodate the new above threshold states.

In our analysis, we use a semi-relativistic Hamiltonian of the form

$$
\begin{aligned}
H & =2 \sqrt{\vec{p}^{2}+m^{2}}+A r-\frac{4 \alpha_{S}}{3 r}\left(1+\frac{\alpha_{S}}{3 \pi}\left(12-n_{f}\right)\left(\ln (\mu r)+\gamma_{E}\right)\right)+V_{S}+V_{L} \\
& =H_{0}+V_{S}+V_{L},
\end{aligned}
$$

where $\mu$ is the renormalization scale, $V_{L}$ contains the $v^{2} / c^{2}$ corrections to the confining potential and the short distance potential is $V_{S}=V_{H F}+V_{L S}+V_{T}+V_{S I}$, with

$$
\begin{aligned}
V_{H F}= & \frac{32 \pi \alpha_{S} \vec{S}_{1} \cdot \vec{S}_{2}}{9 m^{2}}\left\{\left[1-\frac{\alpha_{S}}{12 \pi}(26+9 \ln 2)\right] \delta(\vec{r})\right. \\
& \left.-\frac{\alpha_{S}}{24 \pi^{2}}\left(33-2 n_{f}\right) \nabla^{2}\left[\frac{\ln \mu r+\gamma_{E}}{r}\right]+\frac{21 \alpha_{S}}{16 \pi^{2}} \nabla^{2}\left[\frac{\ln m r+\gamma_{E}}{r}\right]\right\}
\end{aligned}
$$




$$
\begin{aligned}
V_{L S}= & \frac{2 \alpha_{S} \vec{L} \cdot \vec{S}}{m^{2} r^{3}}\left\{1-\frac{\alpha_{S}}{6 \pi}\left[\frac{11}{3}-\left(33-2 n_{f}\right) \ln \mu r+12 \ln m r-\left(21-2 n_{f}\right)\left(\gamma_{E}-1\right)\right]\right\} \\
V_{T}= & \frac{4 \alpha_{S}\left(3 \overrightarrow{S_{1}} \cdot \hat{r} \vec{S}_{2} \cdot \hat{r}-\vec{S}_{1} \cdot \overrightarrow{S_{2}}\right)}{3 m^{2} r^{3}}\left\{1+\frac{\alpha_{S}}{6 \pi}\left[8+\left(33-2 n_{f}\right)\left(\ln \mu r+\gamma_{E}-\frac{4}{3}\right)\right.\right. \\
& \left.\left.-18\left(\ln m r+\gamma_{E}-\frac{4}{3}\right)\right]\right\} \\
V_{S I}= & \frac{4 \pi \alpha_{S}}{3 m^{2}}\left\{\left[1-\frac{\alpha_{S}}{2 \pi}(1+\ln 2)\right] \delta(\vec{r})-\frac{\alpha_{S}}{24 \pi^{2}}\left(33-2 n_{f}\right) \nabla^{2}\left[\frac{\ln \mu r+\gamma_{E}}{r}\right]-\frac{7 \alpha_{S} m}{6 \pi r^{2}}\right\}
\end{aligned}
$$

Using the variational procedure described in Ref. [8], we fit the experimental data for the the charm and $\Upsilon$ systems by varying the parameters $A, \alpha_{S}, m, \mu$ and $f_{V}$, the fraction of vector coupling in the scalar-vector mixture of the confining potential, to find a minimum in $\chi^{2}$. This was done in two ways: first by treating $V_{L}+V_{S}$ as a perturbation and second by treating the entire Hamiltonian non-perturbatively. The results are shown in Table 1.

TABLE 1. Fitted Parameters

\begin{tabular}{lcccc}
\hline & $c \bar{c}$ Pert & $c \bar{c}$ Non-pert & $b \bar{b}$ Pert & $b \bar{b}$ Non-pert \\
\hline$A\left(\mathrm{GeV}^{2}\right)$ & 0.168 & 0.175 & 0.170 & 0.186 \\
\hline$\alpha_{S}$ & 0.331 & 0.361 & 0.297 & 0.299 \\
\hline$m_{q}(\mathrm{GeV})$ & 1.41 & 1.49 & 5.14 & 6.33 \\
\hline$\mu(\mathrm{GeV})$ & 2.32 & 1.07 & 4.79 & 3.61 \\
\hline$f_{V}$ & 0.00 & 0.18 & 0.00 & 0.09 \\
\hline
\end{tabular}

\section{RESULTS AND CONCLUSIONS}

Our results ${ }^{1}$ for the fit to the $c \bar{c}$ spectrum and the predicted $E 1$ transition rates from the resulting wave functions are comparable to recent results for charmonium [6, 7], with the non-perturbative treatment yielding the best fit. The non-perturbative results ${ }^{1}$ for the $b \bar{b}$ spectrum and decays are quite reasonable, though not as good as those from the perturbative treatment. In Table 2 , we show the fit to the $b \bar{b}$ spectrum for the case of the perturbative treatment of $V_{L}+V_{S}$, and in Table 3, we show our predictions for the observed $E 1$ transitions and the for the $E 1$ decays associated with the $\Upsilon\left(1^{3} D_{2}\right)$.

Aside from the above threshold states in $c \bar{c}$, where mixing as well as continuum effects must be included to describe the $X(3872)$ and the $X(3943)$, both treatments of $c \bar{c}$ and $b \bar{b}$ yield very good overall fits. It is striking that for both systems the perturbative fits require the confining terms to be pure scalar, while the non-perturbative fits require a small amount of vector exchange.

\footnotetext{
${ }^{1}$ See: http://www.panic05.lanl.gov/sessions_by_date.php\#sessions3
} 
TABLE 2.

\begin{tabular}{llllll}
\hline & Pert & Expt & & Pert & Expt \\
\hline$\eta_{b}(1 S)$ & 9411.6 & $9300 \pm 28$ & $\eta_{b}(3 S)$ & 10339.5 & \\
\hline$\Upsilon(1 S)$ & 9459.5 & $9460.3 \pm 0.26$ & $\Upsilon(3 S)$ & 10359.5 & $10355.2 \pm 0.5$ \\
\hline $1 \chi_{b 0}$ & 9862.5 & $9859.44 \pm 0.52$ & $3 \chi_{b 0}$ & 10511.6 & \\
\hline $1 \chi_{b 1}$ & 9893.2 & $9892.78 \pm 0.40$ & $3 \chi_{b 1}$ & 10534.5 & \\
\hline $1 \chi_{b 2}$ & 9914.0 & $9912.21 \pm 0.17$ & $3 \chi_{b 2}$ & 10549.8 & \\
\hline $1 h_{b}$ & 9902.1 & & $3 h_{b}$ & 10540.9 & \\
\hline$\eta_{b}(2 S)$ & 9996.5 & & $1{ }^{3} D_{1}$ & 10149.8. & \\
\hline$\Upsilon(2 S)$ & 10020.9 & $10023.26 \pm 0.31$ & $1^{3} D_{2}$ & 10157.6 & $10161.1 \pm 1.7$ \\
\hline $2 \chi_{b 0}$ & 10228.9 & $10232.5 \pm 0.6$ & $1^{3} D_{3}$ & 10163.5 & \\
\hline $2 \chi_{b 1}$ & 10254.0 & $10255.46 \pm 0.55$ & $1{ }^{1} D_{2}$ & 10158.9 & \\
\hline $2 \chi_{b 2}$ & 10270.8 & $10268.65 \pm 0.55$ & & & \\
\hline $2 h_{b}$ & 10261.1 & & & & \\
\hline
\end{tabular}

TABLE 3.

\begin{tabular}{lcclcc}
\hline$\Gamma_{\gamma}(E 1)(\mathrm{keV})$ & Pert & Expt & $\Gamma_{\gamma}(E 1)(\mathrm{keV})$ & Pert & Expt \\
\hline$\Upsilon(2 S) \rightarrow \gamma 1 \chi_{b 0}$ & 1.12 & $1.16 \pm 0.15$ & $\Upsilon(3 S) \rightarrow \gamma 2 \chi_{b 0}$ & 1.64 & $1.30 \pm 0.20$ \\
\hline$\Upsilon(2 S) \rightarrow \gamma 1 \chi_{b 1}$ & 1.79 & $2.11 \pm 0.20$ & $\Upsilon(3 S) \rightarrow \gamma 2 \chi_{b 1}$ & 2.61 & $2.78 \pm 0.43$ \\
\hline$\Upsilon(2 S) \rightarrow \gamma 1 \chi_{b 2}$ & 1.76 & $2.19 \pm 0.20$ & $\Upsilon(3 S) \rightarrow \gamma 2 \chi_{b 2}$ & 2.59 & $2.89 \pm 0.50$ \\
\hline $2 \chi_{b 1} \rightarrow \gamma \Upsilon\left(1^{3} D_{2}\right)$ & 1.47 & & $2 \chi_{b 2} \rightarrow \gamma \Upsilon\left(1^{3} D_{2}\right)$ & 0.47 & \\
\hline$\Upsilon\left(1^{3} D_{2}\right) \rightarrow \gamma 1 \chi_{b 1}$ & 19.7 & & $\Upsilon\left(1^{3} D_{2}\right) \rightarrow \gamma 1 \chi_{b 2}$ & 5.16 & \\
\hline
\end{tabular}

\section{ACKNOWLEDGMENTS}

This research was supported in part by the National Science Foundation under Grant PHY-0244789.

\section{REFERENCES}

1. J. Pumplin, W. W. Repko and A. Sato, Phys. Rev. Lett. 35, 1538 (1975).

2. H. J. Schnitzer, Phys. Rev. Lett. 35, 1540 (1975).

3. E. Eichten, K. Gottfried, T. Kinoshita, K. D. Lane, and T. M. Yan, Phys. Rev. D 17, 3090 (1978).

4. S. N. Gupta, S. F. Radford and W. W. Repko, Phys. Rev. D 26, 3305 (1982).

5. S. N. Gupta, J. M. Johnson, W. W. Repko and C. J. Suchyta III, Phys. Rev. D 49, 1551 (1994).

6. E. Eichten, K. Lane and C. Quigg, Phys. Rev. D 69, 094019 (2004); arXiv:hep-ph/0511179.

7. T. Barnes and S. Godfrey, Phys. Rev. D 72, 054026 (2005) arXiv:hep-ph/0505002. 
8. S. F. Radford and W. W. Repko, Int. J. Mod. Phys. A20, 3774 (2005) arXiv:hep-ph/0409290. 